\title{
AVALANCHES, SANDPILES AND TUTTE DECOMPOSITION
}

Andrei GABRIELOV

\author{
Department of Geology, Cornell University, Ithaca, NY 14853
}

E-mail: andrei@geology.cornell.edu

\section{Received December 10, 1992}

\begin{abstract}
Sandpile and avalanche models of failure were introduced recently (Bak et al., 1987, and an avalanche of publications with references to this paper) to simulate processes of different nature (earthquakes, charge density waves, forest fires, etc., including economics) characterized by self-organized critical behavior. Statistical properties of an important class of these models, Abelian sandpiles (Dhar, 1990) and Abelian avalanches (Gabrielov, 1992), can be investigated analytically due to an Abelian group acting on the phase space. It is shown that the distribution of avalanches in a discrete, stochastic Abelian sandpile model is identical to the distribution of avalanches in a continuous, deterministic Abelian avalanche model with the same redistribution matrix and loading rate vector. For a symmetric redistribution matrix, recurrent formulas for the distribution of avalanches in the Abelian avalanche model lead to explicit expressions containing invariants of graphs known as Tutte polynomials. In general case, an analogue of the Tutte decomposition is suggested for matrices and directed graphs, and the corresponding expressions for the distribution of avalanches in terms of directed tree numbers of a directed graph are found. New combinatorial identities for graphs and directed graphs are derived from these formulas.
\end{abstract}

Abelian avalanche models. An Abelian avalanche model is defined by a finite set $V$ of sites and by a redistribution matrix $\Delta$ with indices in $V$,

$$
\Delta_{i i}>0, \text { for all } i ; \quad \Delta_{i j} \leq 0, \text { for } j \neq i \text {. }
$$

At every site $i$, a value $h_{i}$, the height at $i$, is defined. A vector $\mathbf{h}=\left\{h_{i}, i \in V\right\}$ is called a configuration of the model. The dynamics of the model is defined by a loading rate vector $\mathbf{v}=\left\{v_{i}, i \in V\right\}$ with non-negative components and by a set of thresholds $H_{i}, i \in V$. A site $i$ is stable if $h_{i}<H_{i}$, and a configuration $\mathbf{h}=\left\{h_{i}\right\}$ is stable when $h_{i}<H_{i}$, for all $i$.

A stable configuration evolves in time according to the rule

$$
d \mathbf{h} / d t=\mathbf{v}
$$


An unstable site $i$ breaks, i.e. the heights are redistributed according to the rule

$$
h_{j} \rightarrow h_{j}-\Delta_{i j}, \text { for all } j,
$$

or $\mathbf{h} \rightarrow \mathbf{h}-\delta_{i}$, where $\delta_{i}=\left(\Delta_{i 1}, \ldots, \Delta_{i N}\right)$ is the $i$-th row vector of $\Delta, N$ is the number of sites. If after this break there are other unstable sites, they also break according to the rule (2), until every site becomes stable. This sequence of breaks is called an avalanche. The number of breaks during an avalanche is called its size.

The value $s_{i}=\sum_{j} \Delta_{i j}$ is called the dissipation at the site $i$, and the value $s_{j}^{\prime}=\sum_{i} \Delta_{i j}$ is called the codissipation at the site $j$. It is usually supposed that all the dissipation values are non-negative. For the combinatorial computations of this paper, it is often more natural to suppose that the codissipation values are non-negative at all sites. These two conditions coincide for a symmetric matrix $\Delta$.

Let $\Delta_{0}=\Delta-\mathbf{s}^{\prime}$ where $\mathbf{s}^{\prime}$ is a diagonal matrix with $\mathbf{s}_{i i}^{\prime}=s_{i}^{\prime}$. A directed graph $\Gamma=\Gamma(\Delta)$ is defined by the set of vertices $V(\Gamma)=V$ and an edge drawn from $i$ to $j$ when $\Delta_{i j}<0$. The matrix $\Delta_{0}$ coincides with the Kirchhoff matrix of $\Gamma$ with conductances $-\Delta_{i j}$ (Tutte, 1984, p.138).

A model is called weakly dissipative if all the dissipation values are non-negative and the graph $\Gamma(\Delta)$ has no non-dissipative sink components, i.e. for every subset $W \subseteq V$ with $s_{k}=0$, for all $k \in W$, there exist $i \in W$ and $j \notin W$ with $\Delta_{i j}<0$.

A model is called weakly codissipative if all the codissipation values are non-negative and the graph $\Gamma(\Delta)$ has no non-codissipative source components, i.e. for every subset $W$ of $V$ with $s_{k}^{\prime}=0$, for all $k \in W$, there exist $j \in W$ and $i \notin W$ with $\Delta_{i j}<0$.

It can be shown that each of these two conditions guarantee that every avalanche in the model is finite, i.e. from any configuration we arrive at a stable configuration after a finite number of breaks.

A model is called properly loaded if the graph $\Gamma(\Delta)$ has no non-loaded source components, i.e. for every subset $W$ of $V$ with $v_{k}=0$, for all $k \in W$, there exist $j \in W$ and $i \notin W$ with $\Delta_{i j}<0$. For a properly loaded model, rate of breaks at every site is positive. 
The threshold values $H_{i}$ are not relevant for the dynamics of the model. It is convenient to take $H_{i}=\Delta_{i i}$, for all $i$. Then $h_{i} \geq 0$ if the site $i$ has been broken at least once. Accordingly, we shall consider only non-negative values of $h_{i}$, and define the set of stable configurations $\mathcal{S}$ as

$$
\mathcal{S}=\left\{0 \leq h_{i}<\Delta_{i i}, \text { for all } i\right\}
$$

Let $\mathbf{r}=\left\{r_{i}, i \in V\right\}$ be the vector of the break rates per unit time.

Proposition (Dhar, 1990). $\Delta^{\prime} \mathbf{r}=\mathbf{v}$ where $\Delta^{\prime}$ is the transpose of $\Delta$.

This implies that $\Delta^{\prime}\left(\mathbf{R}_{+}^{V}\right) \supset \mathbf{R}_{+}^{V}$ where $\mathbf{R}_{+}^{V}=\left\{h_{i} \geq 0\right.$, for $\left.i \in V\right\}$. In particular, $\operatorname{det}(\Delta) \neq 0$. We have also $\operatorname{det}(\Delta)>0$ because all the weakly dissipative (or weakly codissipative) matrices constitute a convex domain of non-degenerate matrices containing the unit matrix.

Abelian properties. The following properties (Dhar, 1990; Bjorner and Lovasz, 1991) play the principal role in the dynamics of our models.

Theorem 1. The stable configuration after an avalanche depends only on the starting configuration of the avalanche, and does not depend on the possible choice of the order of breaks during the avalanche.

Hence an avalanche operator $\mathcal{A}: \mathbf{R}_{+}^{V} \rightarrow \mathcal{S}$ is defined. For any vector $\mathbf{u}$ with nonnegative components, the load-avalanche operator $\mathcal{C}_{\mathbf{u}}$ is defined as follows.

$$
\mathcal{C}_{u}(\mathbf{h})=\mathcal{A}(\mathbf{h}+\mathbf{u})
$$

Theorem 2. For any two vectors $\mathbf{u}, \mathbf{v}$ with non-negative components,

$$
\mathcal{C}_{\mathbf{u}} \cdot \mathcal{C}_{\mathbf{v}}=\mathcal{C}_{\mathbf{u}+\mathbf{v}}
$$

Hence every two load-avalanche operators commute, and the result of a series of loading episodes followed by avalanches is the same as if we do all the loading first, with one large avalanche in the end. 
Recurrent configurations. A configuration $\mathbf{h}$ is called recurrent if there exists an avalanche started at a configuration with arbitrarily large components, passing through $\mathbf{h}$. The set of all stable recurrent configurations is denoted by $\mathcal{R}$.

Theorem 3 (Dhar, 1990). The set $\mathcal{R}$ is a fundamental domain for the lattice $\mathcal{L}$ generated by the vectors $\delta_{i}$, i.e. for every configuration $\mathbf{h}$, there exists precisely one configuration $\mathbf{g} \in \mathcal{R}$ equivalent to $\mathbf{h}$ modulo $\mathcal{L}$.

Corollary. The volume of $\mathcal{R}$ is equal to $\operatorname{det}(\Delta)$, and the dynamics of the model on $\mathcal{R}$ is equivalent to a flow on a torus $\mathbf{R}^{V} / \mathcal{L}$ with the constant rate $\mathbf{r}$. In particular, if $T \mathbf{r}=\mathbf{n}$ where $\mathbf{n}$ is a vector with integer components then every trajectory of the model has a period $T$, otherwise every trajectory is quasiperiodic.

Theorem 4. Let $\mathbf{Q}=\left(\Delta_{11}, \ldots, \Delta_{N N}\right)$ be the diagonal vector of $\Delta$. For every vector $\mathbf{v}=\sum_{i} c_{i} \delta_{i}$ with $c_{i} \geq 0$,

$$
\mathcal{A}(\mathcal{S}+\mathbf{Q}+\mathbf{v})=\mathcal{R} .
$$

Theorem 5. Let $\mathbf{Q}_{\mathbf{n}}=\mathbf{Q}-\Delta^{\prime} \mathbf{n}$ and let $\mathbf{V}_{\mathbf{n}}$ be an open negative octant with the vertex at $\mathbf{Q}_{\mathbf{n}}$. If $\mathbf{n}$ is a vector with integer components at least one of which is positive then $\mathbf{V}_{\mathbf{n}}$ does not contain recurrent configurations.

Theorem 6. For a model with non-negative codissipations, $\mathcal{R}=\mathcal{S} \backslash \cup \mathbf{V}_{\mathbf{n}}$ where the union is taken over all non-zero integer vectors $\mathbf{n}$ with components 0 or 1 .

This theorem is equivalent to the description of $\mathcal{R}$ as the set of those stable configurations which do not contain forbidden subconfigurations (Dhar, 1990). For a subset $W$ of $V$, a subconfiguration $\left\{h_{i}, i \in W\right\}$ is called forbidden if

$$
h_{j}<-\sum_{i \in W \backslash j} \Delta_{i j} \text {, for all } j \in W \text {. }
$$

The proof of Theorem 6 includes the following identity for a determinant of an arbitrary matrix $\Delta$. 


$$
\operatorname{det}(\Delta)=\sum_{l \geq 0}(-1)^{|V|-\left|V_{l}\right|} \sum_{\emptyset=V_{0} \subset \ldots \subset V_{l}} \prod_{j \notin V_{l}} \Delta_{j j} \prod_{i=1}^{l} \prod_{j \in V_{i} \backslash V_{i-1}} \sum_{\nu \in V_{i} \backslash j} \Delta_{\nu j}
$$

Marginally stable recurrent configurations. Let $\mathcal{R}_{i}=\overline{\mathcal{R}} \cap\left\{h_{i}=\Delta_{i i}\right\}$ be the set of recurrent configurations where the avalanches starting with the break at the site $i$ originate. Here $\overline{\mathcal{R}}$ is the closure of $\mathcal{R}$.

Theorem 7. For a model with positive codissipations, the volume of $\mathcal{R}_{i}$ is equal to $\operatorname{det}(\Delta)\left(\Delta^{-1}\right)_{i i}=\operatorname{det}(\Delta(i))$ where $\Delta(i)$ is the $i$-th principal minor of $\Delta$.

Theorem 8. The mean per unit time number of avalanches started at $i$ in the model with positive codissipations is equal to $v_{i}\left(\Delta^{-1}\right)_{i i}$. For the model with non-negative codissipations the same is true if we count every avalanche with the multiplicity equal to the number of breaks of its starting site.

Avalanche models vs. sandpile models. An Abelian sandpile model (Dhar, 1990) is defined in the same way as the Abelian avalanche model, for an integer matrix $\Delta$, except the values of $h_{i}$ are integer, time is discrete, and the loading rate $\mathbf{v}$ is random, with the probability $v_{i}$ to add 1 to the value $h_{i}$ at every time step.

Theorem 9. For every site $i \in V$ and every sequence of non-negative integers $k_{j}, j \in V$, the mean number per time step of avalanches in an Abelian sandpile model started at $i$ and such that every site $j$ breaks $k_{j}$ times during the avalanche, is equal to the mean number per unit time of avalanches with the same property in an Abelian avalanche model with the same redistribution matrix $\Delta$ and the same loading vector $\mathbf{v}$. In case of periodic behavior of the Abelian avalanche model, the average over all periodic trajectories should be taken.

In particular, the distributions of sizes of avalanches in the two types of models are identical. 
Tutte decomposition for symmetric matrices and graphs. For a symmetric matrix

$\Delta$, we define the operation of deletion $D_{i j}^{\mathrm{s}}(\Delta)$ as

$$
\Delta_{i i} \rightarrow \Delta_{i i}+\Delta_{i j}, \Delta_{j j} \rightarrow \Delta_{j j}+\Delta_{i j}, \Delta_{i j} \rightarrow 0, \Delta_{j i} \rightarrow 0 .
$$

The operation of contraction $C_{i j}^{\mathrm{s}}(\Delta)$ is defined as

$$
\Delta_{i i} \rightarrow \Delta_{i i}+\Delta_{j j}+2 \Delta_{i j}, \Delta_{i k} \rightarrow \Delta_{i k}+\Delta_{j k}, \Delta_{k i} \rightarrow \Delta_{k i}+\Delta_{k j}, \text { for } k \neq i, j,
$$

with the $j$-th row and column of $\Delta$ removed. We call these operations the Tutte decomposition of a symmetric matrix $\Delta$.

Theorem 10. For every symmetric matrix $\Delta$,

$$
\operatorname{det}(\Delta)=\operatorname{det}\left(D_{i j}^{\mathrm{s}}(\Delta)\right)-\Delta_{i j} \operatorname{det}\left(C_{i j}^{\mathrm{s}}(\Delta)\right)
$$

Due to the corollary of Theorem 3, for the model with symmetric redistribution matrix $\Delta$ and the loading rate $v_{i}=s_{i}>0$, for all $i$, every trajectory has period 1 , and every site breaks once during this period. Hence a periodic trajectory with $m$ avalanches defines a partition of the set $V$ into subsets $V_{1}, \ldots, V_{m}$ and starting sites $i_{\nu} \in V_{\nu}$, for $\nu=1, \ldots, m$.

Let $X(\Delta)=X\left(\Delta ; V_{1}, \ldots, V_{m} ; i_{1}, \ldots, i_{m}\right)$ be the volume of all periodic trajectories with avalanches $V_{1}, \ldots, V_{m}$ and starting sites $i_{1}, \ldots, i_{m}$.

Lemma. For $i=i_{\nu}$ and $j \in V_{\nu}, j \neq i$,

$$
\frac{X(\Delta)}{s_{i}}=\frac{X\left(D_{i j}^{\mathrm{s}}(\Delta)\right)}{s_{i}}-\Delta_{i j} \frac{X\left(C_{i j}^{\mathrm{s}}(\Delta)\right)}{s_{i}+s_{j}} .
$$

Theorem 11. The volume of all periodic trajectories with $m$ avalanches started at sites $i_{1}, \ldots, i_{m}$ is $s_{i_{1}} \ldots s_{i_{m}} \operatorname{det}\left(\Delta_{i_{1}, \ldots, i_{m}}\right)$, the term with $s_{i_{1}} \cdots s_{i_{m}}$ in the expansion of $\operatorname{det}(\Delta)=$ $\operatorname{det}\left(\Delta_{0}+\mathbf{s}\right)$ in $s_{i}$.

Here $\mathbf{s}$ is the diagonal matrix with $\mathbf{s}_{i i}=s_{i}$ and $\Delta_{i_{1}, \ldots, i_{m}}$ is the minor of $\Delta_{0}$ corresponding to removal of the rows and columns $i_{1}, \ldots, i_{m}$. 
Theorem 12. The volume $f_{m}(\Delta)$ of all periodic trajectories with $m$ avalanches satisfies

$$
f_{m}(\Delta)=f_{m}\left(D_{i j}^{\mathrm{s}}(\Delta)\right)-\Delta_{i j} f_{m}\left(C_{i j}^{\mathrm{s}}(\Delta)\right) .
$$

Theorem 13. $\sum_{m \geq 1} f_{m}(\Delta) z^{m}=\operatorname{det}\left(\Delta_{0}+z \mathbf{s}\right)$.

If the matrix $\Delta$ is integer, we define a graph $G=G(\Delta)$ with the set of vertices $V(G)=V$ and $n_{i j}=-\Delta_{i j}$ edges drawn between $i$ and $j$. The matrix $\Delta_{0}$ is the Laplace matrix of $G$, the operation $D_{i j}^{\mathrm{s}}$ corresponds to the deletion of all edges of $G$ between $i$ and $j$, and the operation $C_{i j}^{\mathrm{s}}$ corresponds to the contraction of all edges of $G$ between $i$ and $j$. These operations constitute the Tutte decomposition of $G$. In particular, the number $T(G)$ of spanning trees of $G$ satisfies (Tutte, 1984, p.40)

$$
T(G)=T\left(D_{i j}^{\mathrm{s}}(G)\right)+n_{i j} T\left(C_{i j}^{\mathrm{s}}(G)\right) .
$$

In general, invariants of graphs satisfying (4) are called Tutte polynomials.

Theorem 14. For an integer symmetric matrix $\Delta$,

$$
\operatorname{det}(\Delta)=\sum_{m \geq 1} \sum_{G_{1}, \ldots, G_{m}}\left[\prod_{\nu=1}^{m} T\left(G_{\nu}\right) \sum_{i \in V\left(G_{\nu}\right)} s_{i}\right] .
$$

Here the second sum is taken over all partitions of $G(\Delta)$ into $m$ induced subgraphs $G_{1}, \ldots, G_{m}$.

Due to Theorem 11, the expression in brackets in (5) is equal to the total volume of periodic trajectories with $m$ avalanches $V\left(G_{1}\right), \ldots, V\left(G_{m}\right)$.

Theorem 15. The mean per unit time number of avalanches in an Abelian avalanche model with an integer symmetric matrix $\Delta$ and $v_{i}=s_{i}>0$, for all $i$, is equal to

$$
\frac{1}{\operatorname{det}(\Delta)} \sum_{m \geq 1} m \sum_{G_{1}, \ldots, G_{m}} \prod_{\nu=1}^{m} s\left(G_{\nu}\right) T\left(G_{\nu}\right) .
$$

Due to Theorem 9, the same is valid for the mean per time step number of avalanches in an Abelian sandpile model. 
Tutte decomposition for non-symmetric matrices and directed graphs. For an arbitrary matrix $\Delta$, we define deletion operation $D_{i j}(\Delta)$ as

$$
\Delta_{i i} \rightarrow \Delta_{i i}+\Delta_{j i}, \Delta_{j i} \rightarrow 0
$$

and contraction operation $C_{i j}(\Delta)$ as

$$
\Delta_{i k} \rightarrow \Delta_{i k}+\Delta_{j k}, \text { for all } k
$$

with the $j$-th row and the $j$-th column removed. We call these operations the Tutte decomposition of a matrix $\Delta$.

Theorem 16. For an arbitrary matrix $\Delta$,

$$
\operatorname{det}(\Delta)=\operatorname{det}\left(D_{i j}(\Delta)\right)-\Delta_{j i} \operatorname{det}\left(C_{i j}(\Delta)\right)
$$

Due to the Corollary from Theorem 3, for the Abelian avalanche model with the loading rate $v_{i}=s_{i}^{\prime}>0$, for all $i$, every trajectory has period 1 , and every site breaks once during this period. Let $X(\Delta)=X\left(\Delta ; V_{1}, \ldots, V_{m} ; i_{1}, \ldots, i_{m}\right)$ be the volume of all periodic trajectories with avalanches $V_{1}, \ldots, V_{m}$ and starting sites $i_{1}, \ldots, i_{m}$.

Lemma. For $i=i_{\nu}$ and $j \in V_{\nu}, j \neq i$,

$$
X(\Delta)=X\left(D_{i j}(\Delta)\right)-\Delta_{j i} X\left(C_{i j}(\Delta)\right)
$$

Theorem 17. The volume of all periodic trajectories with $m$ avalanches started at sites $i_{1}, \ldots, i_{m}$ is $s_{i_{1}}^{\prime} \ldots s_{i_{m}}^{\prime} \operatorname{det}\left(\Delta_{i_{1}, \ldots, i_{m}}\right)$, the term with $s_{i_{1}}^{\prime} \cdots s_{i_{m}}^{\prime}$ in the expansion of $\operatorname{det}(\Delta)=$ $\operatorname{det}\left(\Delta_{0}+\mathbf{s}^{\prime}\right)$ in $s_{i}^{\prime}$.

Here $\mathbf{s}^{\prime}$ is the diagonal matrix with $\mathbf{s}_{i i}^{\prime}=s_{i}^{\prime}$ and $\Delta_{i_{1}, \ldots, i_{m}}$ is the minor of $\Delta_{0}$ corresponding to removal of the rows and columns $i_{1}, \ldots, i_{m}$.

If the matrix $\Delta$ is integer, we define a directed graph $G=G(\Delta)$ with the set of vertices $V(G)=V$ and $n_{i j}=-\Delta_{j i}$ edges drawn from $i$ to $j$ (note inverse order of indices). 
The matrix $\Delta_{0}=\Delta-\mathbf{s}^{\prime}$ is the Laplace matrix of $G$, the operation $D_{i j}$ corresponds to the deletion of all edges of $G$ from $i$ to $j$, and the operation $C_{i j}$ corresponds to the deletion of all edges of $G$ entering $j$ and the contraction of all edges of $G$ from $i$ to $j$, the contracted vertex denoted by $i$. We call these operations a Tutte decomposition of a directed graph $G$.

Theorem 18. For a vertex $k$ of $G$, let $T(G ; k)$ be the number of all spanning trees in $G$ directed from $k$. Then

$$
T(G ; k)=T\left(D_{i j}(G) ; k\right)+n_{i j} T\left(C_{i j}(G) ; k\right) .
$$

In case $k=j$, the vertex $k$ is absent in $C_{i j}(G)$ and $T\left(C_{i j}(G) ; k\right)=0$.

Theorem 19. For an integer matrix $\Delta$,

$$
\operatorname{det}(\Delta)=\sum_{m \geq 1} \sum_{G_{1} \ldots G_{m}} \sum_{i_{1}, \ldots, i_{m}}\left[\prod_{\nu=1}^{m} T\left(G_{\nu} ; i_{\nu}\right) s_{i_{\nu}}^{\prime}\right] .
$$

Here the second sum is taken over all partitions of $G(\Delta)$ into $m$ induced subgraphs $G_{1}, \ldots, G_{m}$, and the third sum is taken over all m-tuples $\left\{i_{\nu} \in V\left(G_{\nu}\right)\right\}$.

Due to Theorem 17, the term in brackets in (6) is equal to the total volume of periodic trajectories with $m$ avalanches $V\left(G_{1}\right), \ldots, V\left(G_{m}\right)$ started at $i_{1}, \ldots, i_{m}$.

Acknowledgements. This work was done when the author was visiting Cornell University, under NSF grant \#EAR-91-04624, and Rutgers University, under NSF grant \#DMS$92-13357$.

\section{REFERENCES}

P. Bak, C. Tang and K. Wiesenfeld, 1987. Phys. Rev. Lett. 59, 381

A. Bjorner, L. Lovasz, 1991. Chip firing games on directed graphs, preprint.

D. Dhar, 1990. Phys. Rev. Lett. 64, 1613.

A. Gabrielov, 1992. Abelian avalanches and Tutte polynomials, preprint. To appear in Physica A.

W.T. Tutte, 1984. Graph Theory. Addison-Wesley Publishing Company. 